\title{
On Outage Performance of Spectrum-Sharing Communication over M-Block Fading
}

\author{
AbdulRahman Alabbasi, Zouheir Rezki, Basem Shihada \\ Computer, Electrical and Mathematical Science and Engineering (CEMSE) Division \\ King Abdullah University of Science and Technology (KAUST) \\ Email: \{abdulrahman.alabbasi, zouheir.rezki, basem.shihada\}@kaust.edu.sa
}

\begin{abstract}
In this paper, we consider a cognitive radio system in which a block-fading channel is assumed. Each transmission frame consists of $M$ blocks and each block undergoes a different channel gain. Instantaneous channel state information about the interference links remains unknown to the primary and secondary users. We minimize the secondary user's targeted outage probability over the block-fading channels. To protect the primary user, a statistical constraint on its targeted outage probability is enforced. The secondary user's targeted outage region and the corresponding optimal power are derived. We also propose two sub-optimal power strategies and derive compact expressions for the corresponding outage probabilities. These probabilities are shown to be asymptotic lower and upper bounds on the outage probability. Utilizing these bounds, we derive the exact diversity order of the secondary user outage probability. Selected numerical results are presented to characterize the system's behavior.
\end{abstract}

Index Terms-Block-Fading channel, outage probability, resource allocation, spectrum-sharing.

\section{INTRODUCTION}

The demand for reliable and real-time communication systems has increased due to the improvement in quality of services of the communication applications, e.g., video streaming for mobile devices [1]. This magnifies the necessity of studying delay-limited performances. It is well known that decoding longer codewords of the received message increases the robustness of the system to noise and interference. However, such decoding increases the receiving delay, which prevents widespread deployment of real-time communication systems. Therefore, studying Block-Fading (BF) models is essential to tackle and characterize delay-limited systems [2]. In BF models, a message is encoded into $M$ codewords, i.e., one frame, each block undergoes different fading. Each codeword is comprised of $N$ symbols that undergo a similar fading gain. The outage probability metric over BF channels is a critical measure for delay-limited systems with fixed communication rate [3]. In addition, investigating the $\mathrm{BF}$ based cognitive radio (CR) system (to improve the system's throughput and energy efficiency [4], [5], [6] through spectrum sharing) while utilizing the outage performance metric is essential to deploy a delay limited and high throughput future communication technologies.

The work in [3] tackles the minimization of the outage probability under long-term power and short-term power constraints with perfect knowledge of the transmitter-receiver channel state information (CSI). In [7], authors addressed the problems of maximizing the ergodic capacity and service outage capacity under CR environment subject to primary user (PU) outage constraints and secondary user (SU) power and outage constraints. They assumed a multi-carrier system, a perfect knowledge of both the PU transmission power policy, and the entire network channel gains. Under no-constraint on the number of decoding blocks, authors of [8] derived optimal power to maximize the ergodic capacity and minimize the outage capacity. This analysis is conducted while protecting the PU by limiting its outage to a certain threshold.

In this work, we consider a CR system with $M$ blockfading channels. The main objective is to minimize the targeted SU's outage probability and to provide the corresponding optimal adaptive power with a minimal outage region. Exact mutual information of the SU is difficult to compute due to interference from PU. Thus, we consider the SU targeted outage probability, defined as the probability that a fixed communication rate is above a lower bound of the mutual information of the SU system. The terms outage and targeted outage are used interchangeably.

Unlike [3], we investigate the impact of the CR environment on system performance limits. Specifically, we consider three constraints: short-term power, long-term power, and CR constraints. The short-term power constraint limits the transmission power over $M$ blocks to a certain threshold. In practice, this constraint ensures that the linear power amplifier does not operate in the saturation region and remains in the linear amplification region. The long-term power constraint forces the expected value of the frame power to be less than a certain threshold. This constraint preserves the transmitter's battery life. The CR constraint represents the effect of secondary transmission on the PU outage probability. We assume that the PU operates in a stringent delay-limited mode and thus decodes its message over one block and decodes interference as noise. We assume that secondary transmitter (ST) and secondary receiver (SR) share the CSI of their instantaneous channel gain, whereas only the statistical CSI of the crosslink between the secondary and the primary channel is made available. These assumptions move the system closer to practical scenarios because SU systems seldom know the CSI and power policies of PU systems. The use of the outage metric also underscores the practicality of the proposed system, since it characterizes the real-time performance of a communication system. 
In the previous setting, we provide the following contributions. First, we provide an optimal power allocation that minimizes SU's targeted outage probability over the $M$ blocks and the corresponding outage region. Second, to overcome the high complexity of the above problem solution we propose sub-optimal power strategies. Third, the corresponding outage probabilities of these sub-optimal strategies are proven to be lower and upper bounds on the targeted outage probability with tractable expressions. Fourth, by utilizing these bounds we obtain the exact diversity order (DO) of the system. Fifth, the sub-optimal power strategy of the asymptotic lower bound is proven to be optimal at high power regime. Finally, selected numerical results show the effect of the CR constraint on the performance yardstick.

Unlike the work in [9] and [7], where a perfect CSI of all network channels is assumed available at ST side, this framework captures a more practical scenario where the crosslink between the ST and the primary receiver (PR) is unknown. Furthermore, the effect of the PU's activity on the SU's outage performance is analytically and numerically captured. We note that due to the length constraint, several details and proofs have been removed from this version of the paper.

The rest of the paper is organized as follows. Section II describes the system model. Section III summarizes a related background to the proposed system. Section IV discusses the SU outage problem formulation and its optimal power allocation. Section V derives upper and lower bounds (suboptimal strategies) on the outage probability and then derives the diversity order of the exact system outage. Numerical results are presented in Section VI.

Notation: The notation used throughout this paper is defined here.

- A bold small letter indicates a vector, i.e., $\mathbf{a}=$ $\left\{a_{1}, a_{2}, \ldots, a_{M}\right\}, M$ is the vector length.

- The operator $\langle$.$\rangle presents the arithmetic mean of its$ argument, i.e., $\langle\mathbf{a}\rangle=\frac{1}{M} \sum_{i=1}^{M} a_{i}$.

- The operator $\mathbb{E}\{$.$\} is the expectation of its argument.$

- $[a]^{+}$denotes $\max (a, 0)$.

- $a<b \Longleftrightarrow c<d$ means that the inequality of the left side of $\Longleftrightarrow$ implies that on the right side.

- It is said that $a \approx b$ if $\lim _{P \rightarrow \infty} \frac{a}{b} \approx 1$.

- The operators $\leq$ and $\geq$ are element wise operators (unless mentioned otherwise), i.e., $\mathbf{a} \geq 0$ means that all elements of vector a are greater or equal to zero.

\section{SySTEM MOdEL}

We consider a two-user CR system in which both users communicate through a block-fading channel. Figure 1 depicts the system model during the $i^{\text {th }}$ SU block and the $j^{\text {th }}$ PU block, $i \in\{1, \ldots, M\}, j \in\{1, \ldots, K\}$, where $M$ and $K$ are the number of blocks in the SU and PU frames, respectively. In Fig. 1, the fading channels between the primary transmitter (PT) and PR, ST and SR, ST and PR, and PT and SR are designated as $h_{p j}, h_{s i}, h_{s p i}$, and $h_{p s i}$, respectively. Note that the corresponding channel gains are the square modulus of the channels, i.e., $\gamma_{p j}=\left|h_{p j}\right|^{2}, \gamma_{s i}=\left|h_{s i}\right|^{2}, \gamma_{s p i}=$

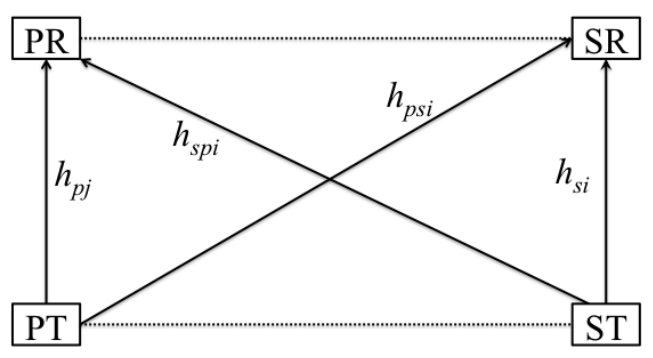

Fig. 1. The system model showing the i-th block of the SU's link, j-th block of the PU's link, and the corresponding cross-links.

$\left|h_{s p i}\right|^{2}, \gamma_{p s i}=\left|h_{p s i}\right|^{2}$. The channel gain vectors are expressed as $\gamma_{p}=\left\{\gamma_{p 1}, \gamma_{p 2}, \ldots, \gamma_{p K}\right\}, \gamma_{s}=\left\{\gamma_{s 1}, \gamma_{s 2}, \ldots, \gamma_{s M}\right\}$, $\gamma_{s p}=\left\{\gamma_{s p 1}, \gamma_{s p 2}, \ldots, \gamma_{s p M}\right\}, \gamma_{p s}=\left\{\gamma_{p s 1}, \gamma_{p s 2}, \ldots, \gamma_{p s K}\right\}$. The channels $h_{p j}, h_{s i}, h_{s p i}$, and $h_{p s j}$ are assumed to be independent random variables with continuous and bounded probability density functions (PDFs) and variances $\sigma_{p}^{2}, \sigma_{s i}^{2}$, $\sigma_{s p}^{2}$, and $\sigma_{p s}^{2}$, respectively. For simplicity, PU is assumed to operate in a stringent delay-limited constraint and thus must encode/decode over a single fading block, i.e., $K=1$.

The received signal at the SR is expressed as follows,

$$
y_{s i}=h_{s i} x_{s i}+h_{p s i} x_{p}+n_{i}, \quad \forall i \in\{1, \ldots, M\},
$$

where $x_{s i}$ and $x_{p}$ are the transmitted secondary and primary signals. The Additive-White-Gaussian-Noise (AWGN) is expressed as $n_{i}$. It is assumed that ST and SR share perfect and casual CSI of $\gamma_{s i}, i=1, \ldots, M$, through a low-rate, error-free, and limited-delay feedback link. We assume a statistical CSI of $\gamma_{s p i}$ at ST. The SU minimizes its outage probability via power adaptation while maintaining a fixed communication rate. It is assumed that PU decodes interference as noise. This is because PU does not have access to the CSI of $\gamma_{s p i}$. It is also assumed that PU's transmission power has a peak constraint, $\mathcal{P}_{p}$. This enables us to consider the worst case scenario of interference toward SU. On the other hand, SU adapts its power with each channel gain, $\gamma_{s i}$ and the effect of PU transmission on $\mathrm{SU}$ is also considered in our framework.

\section{RELATED BACKGROUND}

We briefly describe the minimum outage probability over $M$ fading blocks along with optimal power adaptation derived in [3]. While in [3], authors did not address the communication in a spectrum sharing scenario, it is of interest to give an overview of the results therein to highlight the solution structure. Consider a communication on the secondary network where the primary network is removed. Clearly, the system becomes a point-to-point communication between the secondary transmitter and the secondary receiver.

The objective of problem $\mathfrak{P}_{0}$ is to minimize SU's outage probability over $M$ blocks, subject to two constraints, namely, a short-term and a long-term power constraints. Problem $\mathfrak{P}_{0}$ is defined as follows,

$$
\mathfrak{P}_{0}: \quad \quad \min _{\mathbf{p}\left(\boldsymbol{\gamma}_{s}\right)} \operatorname{Pr}\left[\mathcal{I}_{M}\left(\mathbf{p}\left(\boldsymbol{\gamma}_{s}\right), \boldsymbol{\gamma}_{s}\right)<R_{s}\right]
$$




$$
\begin{array}{ll}
\text { s.t. } & \mathfrak{C}_{1}:\left\langle\mathbf{p}\left(\boldsymbol{\gamma}_{s}\right)\right\rangle \leq \mathcal{P}^{s t} \\
& \mathfrak{C}_{2}: \mathbb{E}\left\{\left\langle\mathbf{p}\left(\boldsymbol{\gamma}_{s}\right)\right\rangle\right\} \leq \mathcal{P}^{l t},
\end{array}
$$

where $\mathcal{I}_{M}\left(\mathbf{p}\left(\boldsymbol{\gamma}_{s}\right), \boldsymbol{\gamma}_{s}\right)=\frac{1}{M} \sum_{i=1}^{M} \frac{1}{2} \log \left(1+p_{i}\left(\gamma_{s i}\right) \gamma_{s i}\right)$ is the SU's mutual information over a single frame. The SU's fixed rate is $R_{s}$. The adaptive transmission power is designated as $\mathbf{p}\left(\gamma_{s}\right)$. The short-term constraint, in (2b), limits the transmission power over one frame to $\mathcal{P}^{s t}$. The long-term constraint, in (2c), enforces the expected value of the transmitted power per frame to $\mathcal{P}^{l t}$. It is assumed that $\mathcal{P}^{l t}<\mathcal{P}^{s t}$. This assumption is intuitively valid given that in the opposite case, i.e., $\mathcal{P}^{l t}>\mathcal{P}^{s t}$, the long-term constraint becomes inactive.

We begin by stating some definitions. Let $\mathcal{Q}$ be the region defined by ordering the channel gains, in a descending order, as: $\mathcal{Q}=\left\{\gamma_{s} \in \mathbb{R}_{+}^{M}: \gamma_{s 1} \geq \cdots \geq \gamma_{s M}\right\}$. A budget power is defined as a combination of both $\mathfrak{C}_{1}$ and $\mathfrak{C}_{2}$ constraints, expressed as follows,

$$
\hat{s}=\min \left(\mathcal{P}^{s t}, s^{*}\right),
$$

where $s^{*}$ is understood as an instantaneous power threshold that reflects the effect of the long-term power constraint, $\mathfrak{C}_{2}$. The value of $s^{*}$ is obtained by solving the following equality,

$$
\int_{\overline{\mathcal{R}}\left(s^{*}\right)}\left\langle\mathbf{p}\left(\boldsymbol{\gamma}_{s}\right)\right\rangle d G\left(\boldsymbol{\gamma}_{s}\right)=\mathcal{P}^{l t},
$$

where $G\left(\gamma_{s}\right)$ is the cumulative density function (CDF) of $\gamma_{s}$, and $\overline{\mathcal{R}}\left(s^{*}\right)$ is the no-outage region defined as follows,

$$
\overline{\mathcal{R}}(s)=\left\{\boldsymbol{\gamma}_{s} \in \mathbb{R}_{+}^{M} ;\left\langle\mathbf{p}\left(\boldsymbol{\gamma}_{s}\right)\right\rangle \leq s\right\} .
$$

The optimal power allocation of $\mathfrak{P}_{0}$ is expressed as follows,

$$
\mathbf{p}^{*}\left(\gamma_{s}\right)=\left\{\begin{array}{lll}
\mathbf{p}^{s t}\left(\gamma_{s}\right) ; & \text { if } & \gamma_{s} \notin \mathcal{U}\left(R_{s}, \hat{s}\right) \\
0 ; & \text { if } & \gamma_{s} \in \mathcal{U}\left(R_{s}, \hat{s}\right)
\end{array},\right.
$$

where $\mathbf{p}^{s t}\left(\gamma_{s}\right)$ has the following water-filling structure,

$$
p_{m}^{s t}\left(\boldsymbol{\gamma}_{s}\right)=\left[\lambda^{s t}\left(\mu, \gamma_{s}\right)-\frac{1}{\gamma_{s m}}\right]^{+} \quad \forall i \in\{1, \ldots, M\},
$$

and the Lagrangian multiplier $\lambda^{s t}\left(\mu, \gamma_{s}\right)=\frac{1}{\mu} \sum_{l=1}^{\mu} \frac{1}{\gamma_{s l}}+\frac{M}{\mu} \hat{s}$, where $\mu$ is the unique integer in $\{1, \ldots, M\}$, such that $\lambda_{s}^{l t}\left(\mu, \gamma_{s}\right) \geq \frac{1}{\gamma_{s m}}$ for $m \leq \mu$ and $\lambda_{s}^{l t}\left(\mu, \gamma_{s}\right)<\frac{1}{\gamma_{s m}}$ for $m>\mu$. The corresponding outage region is designated as $\mathcal{U}\left(R_{s}, \hat{s}\right)$. It is more convenient to show the outage region as the intersection with $\mathcal{V}_{\mu}$, such that,

$$
\begin{aligned}
& \mathcal{U}\left(R_{s}, \hat{s}\right) \cap \mathcal{V}_{\mu}= \\
& \left\{\gamma_{s} \in \mathcal{Q}: \frac{1}{M} \sum_{m=1}^{\mu} \frac{1}{2} \log \left(1+\gamma_{s m} p_{m}^{s t}\left(\gamma_{s}\right)\right)<R_{s}\right\},
\end{aligned}
$$

The region $\mathcal{V}_{\mu}$ is a sub-region of $\mathcal{Q}$, defined such that the corresponding power elements are positive, i.e., $\left\{p_{1}^{s t}\left(\gamma_{s}\right), \ldots, p_{\mu}^{s t}\left(\gamma_{s}\right)\right\}>0$. The outage region in (8) satisfies the minimum fixed rate, whereas both the short-term and longterm power constraints are already satisfied through the design of $p_{m}^{s t}\left(\gamma_{s}\right)$. Note that the outage region, i.e., $\mathcal{U}\left(R_{s}, \hat{s}\right)$, is equal to the complement of the no outage region, i.e., $\mathbb{R}_{+}^{M}-\overline{\mathcal{R}}(\hat{s})$, where the optimal power associated with $\overline{\mathcal{R}}(\hat{s})$ is given in [3].

\section{Minimum Outage Probability in CR Framework}

In this section, the SU's outage probability problem is formulated under the CR environment. The objective of this problem is to minimize the $\mathrm{SU}$ outage probability over $\mathrm{BF}$ channel, subject to three constraints, namely, short-term power constraint, long-term power constraint, and PU outage constraint (CR constraint). Both the short-term and long-term power constraints are described in Section III. The CR constraint manifests the effect of a multi-block SU communication system on a single-block PU communication system. Assuming that PU operates in a delay-limited mode, thus the PU is protected from SU interference by limiting the PU's outage probability to a certain threshold. Due to the interference of PU, in (1), the exact mutual information of the SU is difficult to compute. We therefore consider a lower bound on the mutual information of the SU, i.e., $\mathcal{I}_{M}^{(s)}\left(\mathbf{p}_{s}\left(\gamma_{s}\right), \gamma_{s}\right) \geq$ $\mathcal{I}_{M}^{(s-)}\left(\mathbf{p}_{s}\left(\gamma_{s}\right), \gamma_{s}\right)$, where $\mathbf{p}_{s}\left(\gamma_{s}\right)$ is the SU's transmission power. The expression of $\mathcal{I}_{M}^{(s-)}\left(\mathbf{p}_{s}\left(\gamma_{s}\right), \gamma_{s}\right)$ is derived along similar lines as in [10], i.e.,

$$
\mathcal{I}_{M}^{(s-)}\left(\mathbf{p}_{s}\left(\boldsymbol{\gamma}_{s}\right), \boldsymbol{\gamma}_{s}\right)=\frac{1}{2 M} \sum_{i=1}^{M} \log \left(1+\frac{\mathbf{p}_{s}\left(\boldsymbol{\gamma}_{s}\right) \gamma_{s i}}{1+\mathcal{P}_{p} \sigma_{p s}^{2}}\right) \text {. }
$$

Consequently, the upper bound of the exact outage probability is $P_{\text {out }}^{+}=\operatorname{Pr}\left[\mathcal{I}_{M}^{(s-)}\left(\mathbf{p}_{s}\left(\gamma_{s}\right), \gamma_{s}\right)<R_{s}\right]$. Hereafter, $P_{\text {out }}^{+}$is designated as the outage probability of the SU. Likewise, the exact PU mutual information is difficult to compute. Therefore, we formulate the $\mathrm{CR}$ constraint using a lower bound on the PU mutual information, i.e., $\mathcal{I}_{i}^{(p-)}$. Following similar lines as in [10], given $\gamma_{s i}$ on the SU side, $\mathcal{I}_{i}^{(p-)}$ is obtained as $\mathcal{I}_{i}^{(p-)}=\frac{1}{2} \log \left(1+\frac{\mathcal{P}_{p} \gamma_{p}}{p_{s i}\left(\gamma_{s i}\right) \sigma_{s p}^{2}+1}\right)$. Note that the condition on $\gamma_{s i}$ follows from the fact that we enforce the corresponding PU outage constraint on the SU's side. Recall that $\gamma_{p}$ is known at PU and $\gamma_{s i}$ is known at SU. It follows that the PU's outage probability constraint is upper bounded by $\operatorname{Pr}\left[\mathcal{I}_{i}^{(p-)}<R_{p} \mid \gamma_{s i}\right]$. The fixed rate of PU is designated as $R_{p}$. The proposed problem, $\mathfrak{P}_{1}$, is formulated as follows,

$$
\begin{aligned}
& \mathfrak{P}_{1}: \min _{\mathbf{p}_{s}\left(\boldsymbol{\gamma}_{s}\right)} \operatorname{Pr}\left[\mathcal{I}_{M}^{(s-)}\left(\mathbf{p}_{s}\left(\boldsymbol{\gamma}_{s}\right), \boldsymbol{\gamma}_{s}\right)<R_{s}\right] \\
& \text { s.t. } \mathfrak{C}_{1}:\left\langle\mathbf{p}_{s}\left(\boldsymbol{\gamma}_{s}\right)\right\rangle \leq \mathcal{P}^{s t} \\
& \mathfrak{C}_{2}: \mathbb{E}\left\{\left\langle\mathbf{p}_{s}\left(\boldsymbol{\gamma}_{s}\right)\right\rangle\right\} \leq \mathcal{P}^{l t} \\
& \mathfrak{C}_{3 i}: \operatorname{Pr}\left[\mathcal{I}_{i}^{(p)}<R_{p} \mid \gamma_{s i}\right] \leq \epsilon, \forall i \in\{1, \ldots, M\} .
\end{aligned}
$$

The constant $\epsilon$ is PU's tolerance in terms of its quality of service (QoS) due to the effect of ST's interference. In order to solve $\mathfrak{P}_{1}$, constraint (10d) is converted to an instantaneous power constraint as follows,

$$
\begin{gathered}
\mathfrak{C}_{3 i}: \quad \operatorname{Pr}\left[\frac{1}{2} \log \left(1+\frac{\mathcal{P}_{p} \gamma_{p}}{p_{s i}\left(\gamma_{s i}\right) \sigma_{s p}^{2}+1}\right)<R_{p} \mid \gamma_{s i}\right] \leq \epsilon \\
\Longrightarrow F_{\gamma_{p} \mid \gamma_{s i}}\left(\frac{\left(p_{s i}\left(\gamma_{s i}\right) \sigma_{s p}^{2}+1\right)\left(e^{2 R_{p}}-1\right)}{\mathcal{P}_{p}}\right) \leq \epsilon \\
\Longrightarrow p_{s i}\left(\gamma_{s i}\right) \leq\left[\frac{F_{\gamma_{p} \mid \gamma_{s i}}^{-1}(\epsilon) \mathcal{P}_{p}}{\left(e^{2 R_{p}}-1\right) \sigma_{s p}^{2}}-\frac{1}{\sigma_{s p}^{2}}\right]^{+}=\mathcal{P}_{p u},
\end{gathered}
$$


where (11c) results from the independence between $\gamma_{p}$ and $\gamma_{s i}$, and the fact that $F_{\gamma_{p} \mid \gamma_{s i}}$, being a CDF, is a monotonically non-decreasing function. Note that $\mathcal{P}_{p u}$ increases with both $\epsilon$ and $\mathcal{P}_{p}$, and decreases with $R_{p}$.

The solution of problem $\mathfrak{P}_{1}$ contains the effect of constraint $\mathfrak{C}_{3 i}$ and the interference from PT. The optimal power allocation of $\mathfrak{P}_{1}$ is expressed as follows,

$$
\hat{\mathbf{p}}_{s}\left(\boldsymbol{\gamma}_{s}\right)=\min \left(\mathbf{p}_{s}^{*}\left(\boldsymbol{\gamma}_{s}\right), \mathcal{P}_{p u}\right),
$$

where the function $\min \left(\mathbf{p}_{s}^{*}\left(\gamma_{s}\right), \mathcal{P}_{p u}\right)$ is an element-wise function of $\mathbf{p}_{s}^{*}\left(\gamma_{s}\right)$. The power profile, $\mathbf{p}_{s}^{*}\left(\gamma_{s}\right)$, is expressed as follows,

$$
\mathbf{p}_{s}^{*}\left(\gamma_{s}\right)=\left\{\begin{array}{ll}
\mathbf{p}_{s}^{s t}\left(\gamma_{s}\right) ; & \text { if } \gamma_{s} \notin \mathcal{U}_{c r}\left(R_{s}, \hat{s}\right) \\
0 ; & \text { if } \gamma_{s} \in \mathcal{U}_{c r}\left(R_{s}, \hat{s}\right)
\end{array},\right.
$$

where $\mathbf{p}_{s}^{s t}\left(\boldsymbol{\gamma}_{s}\right)$ is formulated as follows,

$$
p_{s m}^{s t}\left(\gamma_{s}\right)=\left[\lambda_{s}^{s t}\left(\mu, \gamma_{s}\right)-\frac{1+\mathcal{P}_{p} \sigma_{p s}^{2}}{\gamma_{s m}}\right]^{+} \quad \forall i \in 1, \ldots, M .
$$

Recall that $\hat{s}=\min \left(\mathcal{P}^{s t}, s^{*}\right)$ and $s^{*}$ is defined such that,

$$
\int_{\overline{\mathcal{R}_{c r}}\left(s^{*}\right)}\left\langle\mathbf{p}_{s}\left(\gamma_{s}\right)\right\rangle d G\left(\boldsymbol{\gamma}_{s}\right)=\mathcal{P}^{l t},
$$

where $\overline{\mathcal{R}_{c r}}(\hat{s})=\left\{\boldsymbol{\gamma}_{s} \in \mathbb{R}_{+}^{M} ;\left\langle\hat{\mathbf{p}}_{s}\left(\boldsymbol{\gamma}_{s}\right)\right\rangle \leq \hat{s}\right\}$. It is more convenient to define the SU outage region, designated as $\mathcal{U}_{c r}\left(R_{s}, \hat{s}\right)$, as the union of the outage region intersection with each of the positive power regions, i.e., $\bigcup_{\mu=1}^{M} \mathcal{U}_{c r}\left(R_{s}, \hat{s}\right) \cap \mathcal{V}_{\mu}$. Each of these intersections is defined as follows,

$$
\begin{aligned}
& \mathcal{U}_{c r}\left(R_{s}, \hat{s}\right) \cap \mathcal{V}_{\mu}= \\
& \left\{\gamma_{s} \in \mathcal{Q}: \frac{1}{2 M} \sum_{m=1}^{\mu} \log \left(1+\frac{\gamma_{s m} \hat{\mathbf{p}}_{s}\left(\boldsymbol{\gamma}_{s}\right)}{1+\mathcal{P}_{p} \sigma_{p s}^{2}}\right)<R_{s}\right\},
\end{aligned}
$$

where the Lagrangian multiplier $\lambda_{s}^{s t}\left(\mu, \gamma_{s}\right)=$ $\frac{1+\mathcal{P}_{p} \sigma_{p s}^{2}}{\mu} \sum_{l=1}^{\mu} \frac{1}{\gamma_{s l}}+\frac{M}{\mu} \hat{s}$. The region $\mathcal{V}_{\mu}$ is a sub-region of $\mathcal{Q}$, defined such that the corresponding power elements are positive, i.e., $\left\{p_{s 1}^{s t}\left(\boldsymbol{\gamma}_{s}\right), \ldots, p_{s v}^{s t}\left(\boldsymbol{\gamma}_{s}\right)\right\} \geq 0$. The integer $\mu$ is the unique number in $\{1, \ldots, M\}$, such that $\frac{\left(1+\mathcal{P}_{p} \sigma_{p s}^{2}\right)}{\gamma_{s m}} \leq \lambda_{s}^{s t}$ for $m \leq \mu$ and $\frac{\left(1+\mathcal{P}_{p} \sigma_{p s}^{2}\right)}{\gamma_{s m}}>\lambda^{s t}$ for $m>\mu$.

Note that the outage region in (16) differs from the region in (8) by the effect of the CR instantaneous power constraint, $\mathcal{P}_{p u}$, and PT's interference, $\mathcal{P}_{p} \sigma_{p s}^{2}$.

Clearly, the complexity of computing the above outage region and its associated probability increases with the number of blocks, $M$. In a longer version of this work, we have provided a rigorous example of the above analysis for $M=2$ This example shows that the optimal power profile together with the outage regions are tedious to derive, even for $M=2$. Therefore, in the next section, we provide sub-optimal power strategies and their corresponding compact expressions of the outage probability. The associated outage probabilities of these strategies are shown to be lower and upper bounds on $P_{o u t}^{+}$. These bounds are shown to be optimal in a DO sense.

\section{Sub-Optimal Strategies and Diversity Analysis}

As mentioned in the previous section, it is of interest to provide simpler power allocation strategies with relevantly good outage performance. Hence, in the first sub-section below, we derive sub-optimal power strategies and the associated outage probabilities. In the second sub-section, we provide a DO analysis of the CR system.

\section{A. Sub-Optimal Strategies}

The first sub-optimal strategy consists of communication over a single channel block (selection-combining). In this strategy, the power allocated for the entire frame is transmitted in the block with the highest channel gain. The expression of the outage probability of the selection strategy is obtained as follows,

$$
P_{\text {out }}^{u}=\left[F_{\gamma_{s}}\left(\frac{\left(e^{2 M R_{s}}-1\right)\left(1+\mathcal{P}_{p} \sigma_{p s}^{2}\right)}{M \mathcal{P}_{b d}}\right)\right]^{M},
$$

where $F_{\gamma_{s}}$ is the CDF of $\gamma_{s i}$ (since the elements of $\gamma_{s}$ are independent and identicaly distributed (i.i.d.)) and $\mathcal{P}_{b d}$ is defined as follows,

$$
\mathcal{P}_{b d}=\min \left(\mathcal{P}^{s t}, s^{*}, \mathcal{P}_{p u}\right) .
$$

Recall the definition of $s^{*}$ from (15). It is clear that $P_{o u t}^{u}$ decreases with $\mathcal{P}_{b d}$ and increases with $R_{s}$. The expression of the upper bound, $P_{\text {out }}^{u}$, is obtained by replacing the sum of the allocated power over $M$ blocks by the power on the strongest channel gain. A detailed proof of (17) is given in Appendix A. It is noted from [11], that the selection power policy is optimal in the low power regime.

The second sub-optimal strategy distributes the transmission power uniformly over all blocks. It is found that this strategy is optimal in high power regime, i.e., $\mathcal{P}_{b d} \rightarrow \infty$, as stated in the following corollary.

Corollary 1. In high power regime, i.e., $\mathcal{P}_{b d} \rightarrow \infty$, the optimal power allocation of problem $\mathfrak{P}_{1}$ is to uniformly distribute the power over all available $M$ blocks.

Proof: The detailed proof is given in Appendix B.

Considering the optimal power allocation in the high power regime, i.e., $\mathcal{P}_{b d} \rightarrow \infty$, the associated asymptotic lower bound on the outage probability is derived as follows,

$$
P_{\text {out }}^{l, \infty}=\lim _{\mathcal{P}_{b d} \rightarrow \infty} F_{U}\left[\frac{M\left(e^{\left(2 R_{s}\right)}-1\right)\left(1+\mathcal{P}_{p} \sigma_{p s}^{2}\right)}{\mathcal{P}_{b d}}\right],
$$

where $F_{U}=F_{\sum_{i=1}^{M} \gamma_{s i}}$ is the the CDF of the variable $U=$ $\sum_{i=1}^{M} \gamma_{s i}$, i.e., the sum of the SU channel gains over $M$ blocks. The detailed proof of (19) is given in Appendix C.

\section{B. Diversity Analysis}

In this sub-section, we investigate the DO of the proposed system in problem $\mathfrak{P}_{1}$. We begin by investigating the effect of the system's constraints on the budget power. The sub-optimal strategies, proposed in the previous section, are then utilized to derive the DO of the proposed system. In this sub-section, it 
is assumed that all the channel gains, i.e., $\gamma_{s}$ and $\gamma_{p s}$, follow an exponential distribution.

To analyze the DO performance, we study the impact of the system constraints on the budget power of SU as defined in (18). It is known that the diversity analysis is performed at budget power approaches infinity, $\mathcal{P}_{b d} \rightarrow \infty$. Thus, hereafter, we investigate each of the effective parameters on $\mathcal{P}_{b d}$, since the power budget $\mathcal{P}_{b d} \rightarrow \infty$ iff all the elements $\mathcal{P}^{s t}, s^{*}$, and $\mathcal{P}_{p u}$ approach infinity. On the other hand, if one constraint approaches infinity while one or both others are finite constants, then this constraint becomes inactive. First, obviously, $\mathcal{P}^{s t}$ directly affects $\mathcal{P}_{b d}$, with no dependence on other system parameters, i.e., if $\mathcal{P}^{s t}$ is the only active constraint, then increasing $\mathcal{P}^{s t}$ increases the budget power. Second, we observe that $\mathcal{P}_{p u} \rightarrow \infty$ when $\epsilon \rightarrow 1$ : PU has a high tolerance for $\mathrm{SU}$ interference, $R_{p} \rightarrow 0$ : PU is operating under a very low-rate constraint, and $\mathcal{P}_{p} \rightarrow \infty$ : PU has a high power budget. Third, we consider the dependence between $s^{*}$ and $\mathcal{P}^{l t}$ as observed in (15). This dependence is summarized in the following corollary.

Corollary 2. The threshold $s^{*}$ is related to $\mathcal{P}^{l t}$ as follows,

$$
\mathcal{P}^{l t} \rightarrow \infty \Longleftrightarrow s^{*} \rightarrow \infty .
$$

Proof: The proof is given in Appendix D.

Utilizing the upper bound and the asymptotic lower bound derived in (17) and (19), respectively, and considering the effect of the system parameters on $\mathcal{P}_{b d}$, the DO analysis is summarized in the following corollary.

Corollary 3. The DO of the $S U$ outage probability is obtained as $d_{\text {out }}=-\lim _{\mathcal{P}_{\text {bd }} \rightarrow \infty} \frac{\log \left(P_{\text {out }}\right)}{\log \left(\mathcal{P}_{\text {bd }}\right)}=M$.

Proof: A sketch of the SU DO, $d_{\text {out }}$, proof is given as follows. We begin by investigating the corresponding DO of $P_{\text {out }}^{+}$. This is done by analyzing the DO of the upper and lower bounds of $P_{\text {out }}^{+}$, by considering the sub-optimal strategies proposed in the previous sub-section. Then, we compare the obtained DO of $P_{\text {out }}^{+}$with the DO of a similar system but without interference from PU, i.e., the lower bound on the exact outage probability, not $P_{\text {out }}^{+}$. This comparison leads to the exact DO of the system.

The DO of $P_{\text {out }}^{u}$, called $d_{l}^{-}$, is derived using the above definition, i.e., $d_{\text {out }}=-\lim _{\mathcal{P}_{\text {bd }} \rightarrow \infty} \frac{\log \left(P_{\text {out }}\right)}{\log \left(\mathcal{P}_{b d}\right)}$, while replacing $P_{\text {out }}$ by $P_{\text {out }}^{u}$. The DO, $d_{l}^{-}$, is derived as follows,

$d_{l}^{-}=-\lim _{\mathcal{P}_{b d} \rightarrow \infty} \frac{\log \left[1-e^{(-C)}\right]^{M}}{\log \left(\mathcal{P}_{b d}\right)} \approx-\lim _{\mathcal{P}_{b d} \rightarrow \infty} \frac{M \log [C]}{\log \left(\mathcal{P}_{b d}\right)}=M$,

where $C=\frac{\left(e^{\left(2 M R_{s}\right)}-1\right)\left(1+\mathcal{P}_{p} \sigma_{p s}^{2}\right)}{M \mathcal{P}_{b d}}$. The approximation is derived by Taylor expansion of the exponential function in the previous step. Note that the term inside the exponential $\frac{\left(e^{\left(2 M R_{s}\right)}-1\right)\left(1+\mathcal{P}_{p} \sigma_{p s}^{2}\right)}{M \mathcal{P}_{b d}} \rightarrow 0$ as $\mathcal{P}_{b d} \rightarrow \infty$.

The DO of the outage probability in (19), called $P_{\text {out }}^{l, \infty}$, is obtained as follows,

$d_{u, \infty}^{-}=\lim _{\mathcal{P}_{b d} \rightarrow \infty}-\frac{1}{\log \left(\mathcal{P}_{b d}\right)} \log \left(\frac{\gamma(M, z)}{\Gamma(M)}\right)$

$$
\begin{aligned}
& =\lim _{\mathcal{P}_{b d} \rightarrow \infty}-\frac{1}{\log \left(\mathcal{P}_{b d}\right)} \log \left[\frac{(z)^{M}}{(M-1) !} \sum_{n=0}^{\infty}\left(\frac{(-1)^{n}(z)^{n}}{(M+n) n !}\right)\right] \\
& \leq \lim _{\mathcal{P}_{b d} \rightarrow \infty}-\frac{M \log (z)-\log (M !)}{\log \left(\mathcal{P}_{b d}\right)}=M,
\end{aligned}
$$

where $z=\frac{M\left(e^{\left(2 R_{s}\right)}-1\right)\left(1+\mathcal{P}_{p} \sigma_{p s}^{2}\right)}{\mathcal{P}_{b d}}$, the function $\gamma(M, z)=$ $\int_{0}^{z} t^{M-1} e^{-t} d t$ is the lower incomplete Gamma function, and $\Gamma(M)=(M-1)$ ! is the Gamma function. Equality (22a) is obtained by observing that the sum of exponential random variables follows an Erlang distribution with parameter $\lambda=1$ and shape parameter $k=M$ (this is a special case of Chisquare distribution with $2 M$ degree of freedom). The equality (22b) follows by applying Taylor expansion to the lower incomplete Gamma function.

By comparing (21) and (22), we note that, $M=d_{l}^{-} \leq$ $d_{\text {out }}^{-} \leq d_{u, \infty}^{-}=M$, thus,

$$
d_{\text {out }}^{-}=M .
$$

It is known that the DO does not improve by introducing interference to the SU system. We therefore consider the DO of a point-to-point communication system in [3] (noted as $d_{\text {out }}^{+}$) as an upper bound to the exact outage probability of the proposed system, not $P_{o u t}^{+}$. It is found that,

$$
d_{\text {out }} \leq d_{\text {out }}^{+}=M \text {. }
$$

Comparing (23) and (24), we note that the exact DO of the proposed system is bounded as $d_{\text {out }}^{-} \leq d_{\text {out }} \leq d_{\text {out }}^{+}$, thus, $d_{\text {out }}=M$. This concludes the proof of corollary 3 .

\section{NUMERICAL EVALUATION}

In this section, the outage probability of the $\mathrm{SU}\left(P_{\text {out }}^{+}\right)$ and the corresponding outage region over two communication blocks are evaluated. The outage region corresponding to the derived analytical expressions of communication over two blocks, $M=2$ is shown. Numerical analysis compares $P_{\text {out }}^{+}$ to the upper and lower bounds formulas given by (17) and (19), respectively. Herein, all the channel gains, $\gamma_{s i}, \gamma_{p}, \gamma_{p s i}$, and $\gamma_{s p i}$ follows an exponential distribution.

TABLE I SIMULATION PARAMETERS

\begin{tabular}{|l|l|}
\hline Parameter Name & Value \\
\hline Wireless channels & $\begin{array}{l}\text { Rayleigh, } \\
\text { Slow Flat Fading }\end{array}$ \\
\hline \# Coherence Blocks $(M)$ & 1,2 \\
\hline $\mathcal{P}_{p}$ & $10 \mathrm{~dB}$ \\
\hline$P^{s t}$ & $10 \mathrm{~dB}$ \\
\hline$P^{l t}$ & $15 \mathrm{~dB}$ \\
\hline$\epsilon$ & 0.1 \\
\hline Secondary Rate $\left(R_{s}\right)$ & 0.5 bits / symbol \\
\hline Primary Rate $\left(R_{p}\right)$ & 0.5 bits / symbol \\
\hline$\sigma_{s p}^{2}, \sigma_{p s}^{2}$ & 1 \\
\hline
\end{tabular}

Figure 2 shows the outage region, derived in (16), with $M=$ 2 . It is observed that the outage region decreases by increasing $\hat{s}$, in (3). The shape of the outage region changes based on the relationship between $\hat{s}$ and $\mathcal{P}_{p u}$. To further illustrate this 
point, when $\hat{s}=8 \mathrm{~dB}$, the $\mathrm{CR}$ constraint is not active because $\hat{s}<\frac{\mathcal{P}_{p u}}{2}$, the shape of the outage region (a) is similar to that in [3]. When $\hat{s}$ increases to $\hat{s}=10 \mathrm{~dB}$ or $12 \mathrm{~dB}$, it is clear that $\mathcal{P}_{p u}>\hat{s}>\frac{\mathcal{P}_{p u}}{2}$. It is noted that the corresponding outage regions, (b) and (c) are not convex unlike the region in (a). Increasing $\hat{s}$ to $16 \mathrm{~dB}$, where $\hat{s}>\mathcal{P}_{p u}$, results in a partial alignment in the outage region (d) with region (e). Finally, it is observed that increasing $\hat{s}$ to a relatively large value (with respect to $\mathcal{P}_{p u}$ ) does not change the outage region shape, as in (e). This shape of the outage region occurred because the active budget power becomes the $\mathcal{P}_{p u}$ for all realizations of the two block channel.

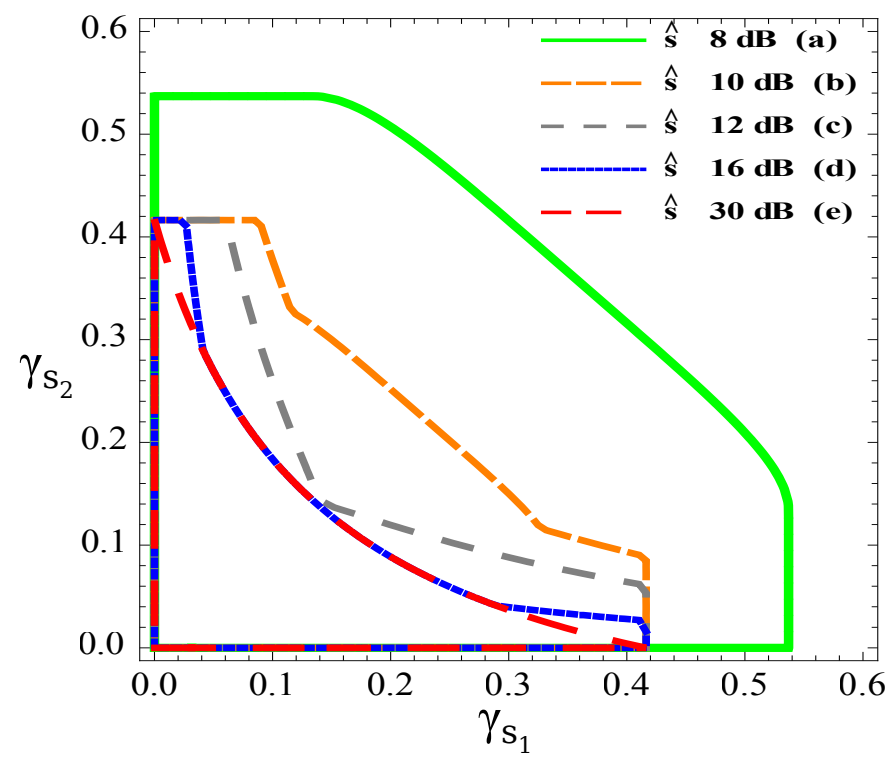

Fig. 2. Outage region for different $\hat{s}, M=2$, and $\mathcal{P}_{p u}=12.1 \mathrm{~dB}$.

Figure 3 shows a comparison among the upper bound $\left(P_{\text {out }}^{u}\right)$ and asymptotically lower bound $\left(P_{\text {out }}^{l, \infty}\right)$ with respect to $P_{\text {out }}^{+}$ for $M=2$. It is observed that the slope of $P_{o u t}^{+}, P_{o u t}^{u}$, and $P_{\text {out }}^{l, \infty}$ is equal to 2 . This supports the results in Corollary 3 . Note that the difference between $P_{\text {out }}^{+}$and $P_{\text {out }}^{u}$ is about $2 \mathrm{~dB}$ at high power regime. It is observed that $P_{\text {out }}^{l, \infty}$ achieves a tight bound to $P_{o u t}^{+}$at high power regime.

\section{CONCLUSION}

In this paper, we considered a spectrum sharing model over a block-fading environment. We minimized the outage probability under several constraints including the primary user outage constraint. We derived the exact expressions of the optimal power allocation and the corresponding outage region. Two sub-optimal power strategies and the corresponding bounds on the outage probability are provided. These bounds are shown to be optimal at high-SNR in the sense that they achieve the same diversity order. Numerical results showed the effect of the cognitive radio constraint on the system performances under all assumptions. We showed that changing the ratio of the cognitive radio constraint to the power constraint affect the convexity of the outage region.

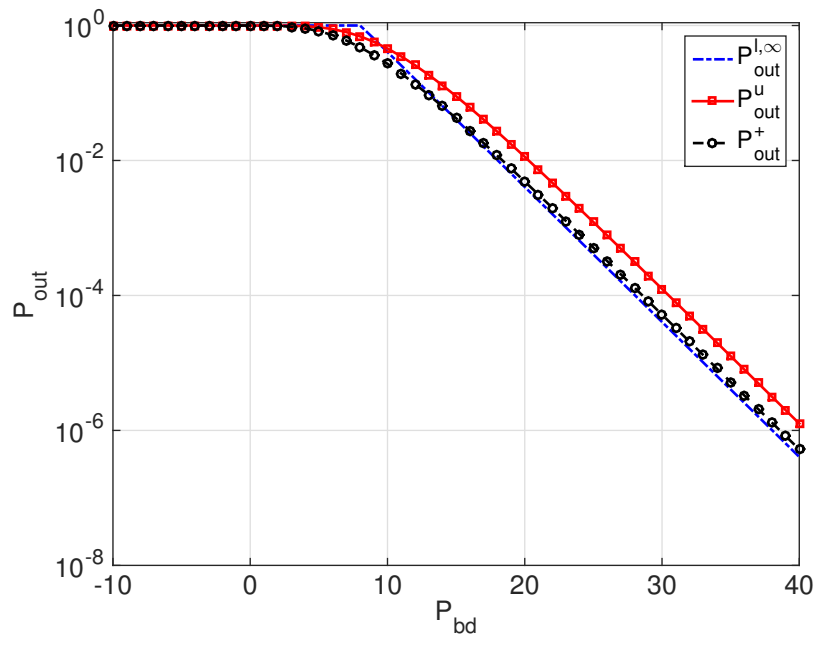

Fig. 3. Comparing the lower and upper bounds with the exact numerical results for $M=2$.

\section{APPENDIX A \\ DERIVATION OF $P_{\text {out }}^{u}$}

The upper bound on $P_{\text {out }}^{+}$is derived by considering the selection-combining over the best fading block. In this scheme, communication is performed over a single block (the one with the strongest channel gain). The corresponding outage probability of the selection-combining scheme is derived as:

$$
\begin{aligned}
P_{\text {out }}^{u} & =\operatorname{Pr}\left[\frac{1}{2 M} \max _{\gamma_{s}} \log \left(1+\frac{M \mathcal{P}_{b d} \gamma_{s i}}{\left(1+\mathcal{P}_{p} \sigma_{p s}^{2}\right)}\right)<R_{s}\right] \\
& =\operatorname{Pr}\left[\max _{\gamma_{s}} \gamma_{s i}<\frac{\left(e^{2 M R_{s}}-1\right)\left(1+\mathcal{P}_{p} \sigma_{p s}^{2}\right)}{M \mathcal{P}_{b d}}\right] \\
& =\left[F_{\gamma_{s}}\left(\frac{\left(e^{2 M R_{s}}-1\right)\left(1+\mathcal{P}_{p} \sigma_{p s}^{2}\right)}{M \mathcal{P}_{b d}}\right)\right]^{M},
\end{aligned}
$$

where (25b) follows from the fact that the logarithm is a monotonically increasing function. Eqaulity (25c) follows because the output of maximum of I.I.D. random variables has the following CDF, $\operatorname{Pr}\left[\max \gamma_{s i} \leq z\right]=\prod_{i=1}^{M} \operatorname{Pr}\left[\gamma_{s i} \leq z\right]=$ $\operatorname{Pr}\left[\gamma_{s} \leq z\right]^{M}$. The function $F_{\gamma_{s}}$ is the CDF of the random variable $\gamma_{s}$. Note that $P_{\text {out }}^{u}$ decreases with $\mathcal{P}_{b d}$ and increases with $R_{s}$.

\section{APPENDIX B}

\section{Asymptotic Optimal Power Assignment}

In this appendix, we prove that the optimal power assignment at high signal-to-noise ratio (SNR) is a uniform assignment over all transmission blocks. Without loss of generality, we prove the optimal power allocation at high SNR for $\mathfrak{P}_{0}$ as follows,

$$
\begin{aligned}
P_{\text {out }} & \stackrel{(a)}{=} \lim _{\mathcal{P}_{b d} \rightarrow \infty} \min _{\mathbf{p}_{s}:\left\langle\mathbf{p}_{s}\right\rangle \leq \mathcal{P}_{b d}} M ! \operatorname{Pr}\left[\bigcup_{\mu=1}^{M}\left[\mathcal{U}\left(R_{s}, \mathcal{P}_{b d}\right) \bigcap \mathcal{V}_{\mu}\right]\right] \\
& =\lim _{\mathcal{P}_{b d} \rightarrow \infty} \min _{\mathbf{p}_{s}:\left\langle\mathbf{p}_{s}\right\rangle \leq \mathcal{P}_{b d}} M ! \operatorname{Pr}\left[\mathcal{Q}_{i}=\mathcal{Q}\right] \sum_{\mu=1}^{M} \operatorname{Pr}[\mu]
\end{aligned}
$$




$$
\begin{gathered}
\operatorname{Pr}\left[\frac{1}{2 M} \sum_{m=1}^{\mu} \log \left(1+\gamma_{s m} p_{s m}^{s t}\left(\gamma_{s}\right)\right)<R_{s} \mid \mu, \mathcal{Q}_{i}=\mathcal{Q}\right] \\
\stackrel{(b)}{=} \lim _{\mathcal{P}_{b d} \rightarrow \infty} \sum_{\mu=1}^{M} \operatorname{Pr}[\mu] \operatorname{Pr}\left[\frac { 1 } { 2 M } \sum _ { m = 1 } ^ { \mu } \operatorname { l o g } \left(1+\gamma_{s m}\right.\right. \\
\left.\left.\left[\frac{1}{\mu} \sum_{l=1}^{\mu} \frac{1}{\gamma_{s l}}+\frac{M}{\mu} \mathcal{P}_{b d}-\frac{1}{\gamma_{s m}}\right]\right)<R_{s} \mid \mu, \mathcal{Q}_{i}\right]
\end{gathered}
$$

where $\mathcal{Q}_{i}$ is defined as the $i^{t h}$ way of sorting $\mathrm{M}$ variables, $i \in\{1, \ldots, M !\}$ and $\operatorname{Pr}\left\{\mathcal{Q}_{i}=\mathcal{Q}\right\}=\frac{1}{M !}$, and region $\mathcal{Q}$ is defined as in section III. Equality (a) is obtained from the outage region definition in (8). The term $M$ ! in (a) represents the number of ways to sort $M$ ! numbers. Equality (b) results from expanding $p_{s m}^{s t}$ as in (14). It is clear that the term within the brackets $\frac{1}{\mu} \sum_{l=1}^{\mu} \frac{1}{\gamma_{s l}}+\frac{M}{\mu} \mathcal{P}_{b d}-\frac{1}{\gamma_{s m}} \approx \frac{M}{\mu} \mathcal{P}_{b d}$ as $\mathcal{P}_{b d} \rightarrow \infty$. It follows that,

$$
\begin{aligned}
P_{\text {out }} & \approx \lim _{\mathcal{P}_{b d} \rightarrow \infty} \sum_{\mu=1}^{M} \operatorname{Pr}[\mu] \operatorname{Pr}\left[\frac{1}{2 M}\right. \\
& \left.\sum_{m=1}^{\mu} \log \left(1+\gamma_{s m}\left[\frac{M}{\mu} \mathcal{P}_{b d}\right]\right)<R_{s} \mid \mu, \mathcal{Q}_{i}\right] \\
& \stackrel{(c)}{\approx} \operatorname{Pr}\left[\frac{1}{2 M} \sum_{m=1}^{M} \log \left(1+\gamma_{s m} \mathcal{P}_{b d}\right)<R_{s} \mid \mu=M, \mathcal{Q}_{i}\right]
\end{aligned}
$$

where (c) follows from the fact that as $\mathcal{P}_{b d} \rightarrow$ $\infty$, all the available blocks are used for transmission $(\mu=M)$. Then, $\lim _{\mathcal{P}_{b d} \rightarrow \infty} \operatorname{Pr}[\mu=M]=$ $\lim _{\mathcal{P}_{b d} \rightarrow \infty} \operatorname{Pr}\left[\frac{1}{M} \sum_{l=1}^{M} \frac{1}{\gamma_{s l}}+\mathcal{P}_{b d} \geq \frac{1}{\gamma_{s M}}\right] \approx 1$.

\section{APPENDIX C \\ ASYMPTOTIC LOWER BOUND ON $P_{\text {out }}^{+}$}

Considering the optimal power allocation in the high power regime, i.e., $\mathcal{P}_{b d} \rightarrow \infty$, the associated asymptotic lower bound of the outage probability is derived as follows [12], [13],

$$
\begin{aligned}
P_{\text {out }}^{+} & \approx \lim _{\mathcal{P}_{b d} \rightarrow \infty} \operatorname{Pr}\left[\frac{1}{2 M} \sum_{m=1}^{M} \log \left(1+\frac{\mathcal{P}_{b d} \gamma_{s i}}{\left(1+\mathcal{P}_{p} \sigma_{p s}^{2}\right)}\right)<R_{s}\right] \quad \text { (28a) } \\
& \geq \lim _{\mathcal{P}_{b d} \rightarrow \infty} \operatorname{Pr}\left[\log \left(1+\frac{1}{M} \sum_{m=1}^{M} \frac{\mathcal{P}_{b d} \gamma_{s i}}{\left(1+\mathcal{P}_{p} \sigma_{p s}^{2}\right)}\right)<2 R_{s}\right] \quad \text { (28b) } \\
& =\lim _{\mathcal{P}_{b d} \rightarrow \infty} \operatorname{Pr}\left[\sum_{i=1}^{M} \gamma_{s i}<\frac{M\left(e^{\left(2 R_{s}\right)}-1\right)\left(1+\mathcal{P}_{p} \sigma_{p s}^{2}\right)}{\mathcal{P}_{b d}}\right]=P_{\text {out }}^{l, \infty} .
\end{aligned}
$$

The asymptotic equivalence at (28a) follows from using the optimal power allocation at high budget power. The inequality (28b) follows from Jensen inequality.

\section{APPENDIX D}

\section{PROOF OF COROLLARY 2}

We begin by proving (20) from left to right. The optimal power solution that achieves minimum outage probability, in (10a), must satisfy the following constraint: $\left\langle\mathbf{p}_{s}\right\rangle \leq s^{*}$.
Therefore, by substituting $\left\langle\mathbf{p}_{s}\right\rangle \leq s^{*}$ in (4), it follows that,

$$
\begin{aligned}
\mathcal{P}^{l t} & =\int_{\overline{\mathcal{R}_{c r}}\left(s^{*}\right)}\left\langle\mathbf{p}_{s}\right\rangle d G\left(\boldsymbol{\gamma}_{s}\right) \leq \int_{\overline{\mathcal{R}_{c r}}\left(s^{*}\right)} s^{*} d G\left(\boldsymbol{\gamma}_{s}\right) \\
& =s^{*} \int_{\overline{\mathcal{R}_{c r}}\left(s^{*}\right)} d G\left(\boldsymbol{\gamma}_{s}\right) .
\end{aligned}
$$

Since the integral in the last term in (29) is a probability, then $0 \leq \int_{\overline{\mathcal{R}}\left(s^{*}\right)} d G\left(\gamma_{s}\right) \leq 1$. It follows that, $s^{*} \geq \mathcal{P}^{l t}$. Hence, it is concluded that $\mathcal{P}^{l t} \rightarrow \infty \Longrightarrow s^{*} \rightarrow \infty$.

To prove the other direction of (20), i.e., $s^{*} \rightarrow \infty \Longrightarrow$ $\mathcal{P}^{l t} \rightarrow \infty$, we assume that $\mathcal{P}_{b d}=s^{*}$; otherwise the longterm constraint will be inactive and this becomes unnecessary to prove. We note that, at $\mathcal{P}_{b d} \rightarrow \infty$, the optimal power is a uniform allocation over all $M$ channels with $p_{s i}\left(\gamma_{s}\right)=\mathcal{P}_{b d}=$ $s^{*}$. The proof of this claim is given in Appendix B. Applying this fact to (4), we obtain,

$$
\begin{aligned}
\mathcal{P}^{l t} & \approx \lim _{\mathcal{P}_{b d} \rightarrow \infty} \mathcal{P}_{b d} \int_{\overline{\mathcal{R}}\left(\mathcal{P}_{b d}\right)} d G\left(\boldsymbol{\gamma}_{s}\right)=\lim _{\mathcal{P}_{b d} \rightarrow \infty} s^{*} \operatorname{Pr}\left[\gamma_{s} \in \overline{\mathcal{R}}\left(\mathcal{P}_{b d}\right)\right] \\
& =s^{*} \lim _{\mathcal{P}_{b d} \rightarrow \infty} \operatorname{Pr}\left[\lambda^{l t}\left(M, \boldsymbol{\gamma}_{s}\right)-\frac{1}{M} \sum_{m=1}^{M} \frac{1}{\gamma_{s m}} \leq \mathcal{P}_{b d}\right] \approx s^{*}
\end{aligned}
$$

It follows that $s^{*} \rightarrow \infty \Longrightarrow \mathcal{P}^{l t} \rightarrow \infty$, which completes the proof of (20).

\section{REFERENCES}

[1] "Voice and video calling over lte (VoLTE)," White Paper, Ericsson, 2014.

[2] A. Alabbasi, Z. Rezki, and B. Shihada, "Energy efficient resource allocation for cognitive radios: A generalized sensing analysis," IEEE Transactions on Wireless Communications, vol. PP, no. 99, pp. 1-1, 2015.

[3] G. Caire, G. Taricco, and E. Biglieri, "Optimum power control over fading channels," IEEE Transactions on Information Theory, vol. 45, no. 5, pp. 1468-1489, 1999.

[4] A. Alabbasi, Z. Rezki, and B. Shihada, "Energy efficient scheme for cognitive radios utilizing soft sensing," in IEEE Wireless Communications and Networking Conference (WCNC), 2014, April 2014, pp. 701-706.

[5] I. F. Akyildiz, W.-Y. Lee, M. C. Vuran, and S. Mohanty, "Next generation/dynamic spectrum access/cognitive radio wireless networks: A survey," Computer Networks, vol. 50, no. 13, pp. 2127 - 2159, 2006.

[6] A. Alabbasi and B. Shihada, "An energy efficient cognitive radio system with quantized soft sensing and duration analysis," in IEEE Wireless Communications and Networking Conference (WCNC), 2015, March 2015, pp. 423-428.

[7] A. Limmanee, S. Dey, and J. Evans, "Service-outage capacity maximization in cognitive radio for parallel fading channels," IEEE Transactions on Communications, vol. 61, no. 2, pp. 507-520, 2013.

[8] X. Kang, R. Zhang, Y.-C. Liang, and H. K. Garg, "Optimal power allocation strategies for fading cognitive radio channels with primary user outage constraint," IEEE Journal on Selected Areas in Communications, vol. 29, no. 2, pp. 374-383, February 2011.

[9] J. Luo, R. Yates, and P. Spasojevic, "Service outage based power and rate allocation for parallel fading channels," IEEE Transactions on Information Theory, vol. 51, no. 7, pp. 2594-2611, 2005.

[10] M. Medard, "The effect upon channel capacity in wireless communications of perfect and imperfect knowledge of the channel," IEEE Transactions on Information Theory, vol. 46, no. 3, pp. 933-946, 2000.

[11] Z. Rezki and M.-S. Alouini, "On the outage capacity of the block fading channel at low-power regime," in IEEE International Symposium on Information Theory (ISIT), 2014, June 2014, pp. 2894-2898.

[12] A. Dembo and O. Zeitouni, Large Deviations Techniques and Applications, ser. Applications of Mathematics.

[13] C. M. Bender and S. A. Orszag, Advanced Mathematical Methods for Scientists and Engineers: Asymptotic methods and perturbation theory. Springer, 1999. 